\title{
An Epidemiological Analysis of the Role of Disease-Induced Root Growth in the Differential Response of Two Cultivars of Winter Wheat to Infection by Gaeumannomyces graminis var. tritici
}

\author{
D. J. Bailey, A. Kleczkowski, and C. A. Gilligan
}

First author: INRA-Agrocampus Rennes, UMR BiO3P, BP 35327, F, 35653 Le Rheu Cedex, France; and second and third authors: Department of Plant Sciences, University of Cambridge, Downing Street, Cambridge, CB2 3EA, U.K. Accepted for publication 15 January 2006.

\begin{abstract}
Bailey, D. J., Kleczkowski, A., and Gilligan, C. A. 2006. An epidemiological analysis of the role of disease-induced root growth in the differential response of two cultivars of winter wheat to infection by Gaeumannomyces graminis var. tritici. Phytopathology 96:510-516.

Epidemiological modeling combined with parameter estimation of experimental data was used to examine differences in the contribution of disease-induced root production to the spread of take-all on plants of two representative yet contrasting cultivars of winter wheat, Ghengis and Savannah. A mechanistic model, including terms for primary infection, secondary infection, inoculum decay, and intrinsic and disease-induced root growth, was fitted to data describing changes in the numbers of infected and susceptible roots over time at a low or high density of inoculum. Disease progress curves were characterized by consecutive phases of primary and secondary infection. No differences in root growth were detected between cultivars in the absence of disease and root production continued for the duration of the experiment. However, significant differences in disease-induced root production were detected between Savannah

and Genghis. In the presence of disease, root production for both cultivars was characterized by stimulation when few roots were infected and inhibition when many roots were infected. At low inoculum density, the transition from stimulation to inhibition occurred when an average of 5.0 and 9.0 roots were infected for Genghis and Savannah, respectively. At high inoculum density, the transition from stimulation to inhibition occurred when an average of 4.5 and 6.7 roots were infected for Genghis and Savannah, respectively. Differences in the rates of primary and secondary infection between Savannah and Genghis also were detected. At a low inoculum density, Genghis was marginally more resistant to secondary infection whereas, at a high density of inoculum, Savannah was marginally more resistant to primary infection. The combined effects of differences in disease-induced root growth and differences in the rates of primary and secondary infection meant that the period of stimulated root production was extended by 7 and 15 days for Savannah at a low and high inoculum density, respectively. The contribution of this form of epidemiological modeling to the better management of take-all is discussed.
\end{abstract}

Over the past three decades, exhaustive searches have detected small differences in the response of certain wheat cultivars to take-all, but useful exploitation of these differences for the purpose of disease control has failed (8). Differences detected in glasshouse tests have not been repeatable under field conditions, where results are notoriously inconsistent (18). The relative magnitude of environmental heterogeneity in field experimentation has largely been blamed for this. It also is possible that empirical methods based on root counts alone, without the benefit of a mechanistic model, are unable to detect the underlying mechanisms that are responsible for differences in disease tolerance. Still, it remains that some wheat cultivars continue to perform better than others in the presence of take-all $(18,19)$. In this article, we identify for the first time some possible epidemiological mechanisms that underlie small differences in host resistance.

Mathematical modeling combined with microcosm experiments performed under carefully controlled environmental conditions offers the opportunity to identify the principal epidemiological features responsible for the differential response of certain wheat cultivars to take-all $(3,4)$. It is now well established that the spread of take-all on wheat depends on primary infection, secondary infection, inoculum decay, and root production $(3,4,6,7$,

Corresponding author: D. J. Bailey; E-mail address: djb21@ cam.ac.uk

DOI: 10.1094/PHYTO-96-0510

(C) 2006 The American Phytopathological Society
10,20). For an epidemic of winter wheat, the initial phase of primary infection occurs as seminal roots grow through the soil and encounter inoculum, and slows progressively as inoculum decays. Depending on the rate of decay of inoculum, primary infection may be restricted largely to the seminal roots. This is followed by an acceleration in the rate of secondary infection on both seminal and adventitious roots, stimulated by the increase in availability of infected roots as a source of inoculum and the availability of susceptible roots for infection as more roots are produced. This means that the rate of secondary infection becomes increasingly dominated by the numbers of infected and susceptible adventitious roots (6).

The availability of susceptible host material for infection is a major determinant for epidemic progress $(11,16)$. For the development of a wheat root system, this can be partitioned into intrinsic and disease-induced root production (4). Intrinsic root production is that which occurs in the absence of a response to disease. Disease-induced root production, in response to infection by Gaeumannomyces graminis var. tritici, long has been implicated in the increased tolerance of certain wheat cultivars to take-all (21). Disease-induced root production in wheat has been clearly demonstrated $(2,22)$ and the precise form of the response analyzed (4), but this form of analysis has yet to be used to compare the extent of the response among different wheat cultivars.

The response is not simple, involving the production of additional adventitious roots at low levels of disease and inhibition of root production when a threshold number of diseased roots is exceeded. In this article, we consider the effect of disease-induced root production as having a direct multiplicative effect on intrinsic 
root production. The key feature of the response function is its nonmonotonic shape, describing a switch from stimulation of root production (for low infection levels) to inhibition (at high levels of infection) (Fig. 1). The form of this response function is a modification of that used in the models of Gilligan et al. (12) and Bailey and Gilligan (4), in that its effect on root growth is multiplicative rather than additive. This allows for more robust treatment of both the stimulation and inhibition phases of the growth response and a more natural interpretation in terms of the relative rate.

This function, relating the growth response to numbers of infected roots per plant can, in turn, be used to define curves describing the period over which the rate of root production is stimulated during the course of an epidemic.

This article deals with the use of epidemiological modeling, in combination with controlled experimentation, as a tool with which to identify, detect, compare, and contrast the response of different wheat cultivars to take-all. We combine the fundamental mechanisms of a take-all epidemic, that is to say, primary infection, secondary infection, inoculum decay, and intrinsic host growth, together with the function for disease-induced root growth, in a simple epidemiological model. We estimate the parameters of the model by fitting to microcosm data and ask the following questions. (i) Do we detect stimulation when few roots are infected and inhibition when many roots are infected for both Genghis and Savannah? (ii) Does the point at which the number of infected roots switches from stimulation to inhibition of root production differ between cultivars? (iii) How does this affect the period of stimulated root growth during the course of an epidemic for each cultivar?

\section{THEORY AND APPROACHES}

Model derivation. Change in infected and susceptible roots over time is described by a set of linked, differential equations that account for the transition from susceptible (S) to infected (I) roots. The generic form of the model is given as

$$
\begin{aligned}
\text { Total roots: } & (\mathrm{d} N / \mathrm{d} t)=b f(N, S, I) g(N) \\
\text { Susceptible roots: } & (\mathrm{d} S / \mathrm{d} t)=b f(N, S, I) g(N)-\Lambda(X, I) S \\
\text { Infected roots: } & (\mathrm{d} I / \mathrm{d} t)=\Lambda(X, I) S \\
\text { Particulate inoculum: } & (\mathrm{d} X / \mathrm{d} t)=-r_{d} X
\end{aligned}
$$

where $f(N, S, I)$ is a response function, $g(N)$ represents a densitydependent intrinsic rate of root growth, and $\Lambda(X, I)$ is the force of infection (1). The model can be further specified by assuming particular forms for the general terms in model 1 to yield

$$
\begin{aligned}
\text { Total roots: } & (\mathrm{d} N / \mathrm{d} t)=b f(I) N(1-[N / \kappa]) \\
\text { Susceptible roots: } & (\mathrm{d} S / \mathrm{d} t)=b f(I) N(1-[N / \kappa])-\left(r_{p} X+r_{s} I\right) S \\
\text { Infected roots: } & (\mathrm{d} I / \mathrm{d} t)=\left(r_{p} X+r_{s} I\right) S \\
\text { Particulate inoculum: } & (\mathrm{d} X / \mathrm{d} t)=r_{d} X \\
\text { Response function: } & f(I)=\left(1+\alpha_{1} I\right) \exp \left(-\alpha_{2} I\right)
\end{aligned}
$$

In the absence of disease, the intrinsic production of new roots increases sigmoidally (starting from the initial value of $N_{0}$ ) during the period of the experiment, and is described in terms of logistic growth, with $\kappa$ representing the maximum number of roots per plant, $b$ the per capita rate of production of susceptible roots, and $N(t)$, the total number of roots at time $t$. Particulate inoculum, $X$, decays exponentially over time with a rate, $r_{d}$. Parameters $b$, $\kappa$, and the initial root number $N_{0}$ (at $t=6$ days) were estimated independently from empirical data for root growth in the absence of disease (we estimated $1 / \kappa$ rather than $\kappa$ for numerical convenience). The decay rate $r_{d}$ was estimated from a separate experiment measuring the decay of particulate inoculum over time. These parameters were fixed at their estimated values before equation 1 was fitted to change in the number of diseased and susceptible roots over time. Rates for primary and secondary infection are given by the parameters $r_{p}$ and $r_{s}$, respectively.

The response function, $f(I)$, is described by a curve which begins with a value of 1.0 when no roots are infected, then increases to a maximum of $f_{\max }=\left(\alpha_{1} / \alpha_{2}\right) \exp \left(1-\alpha_{2} / \alpha_{1}\right)>1$ at $I_{\max }=\left(\alpha_{1}-\right.$ $\left.\alpha_{2}\right) /\left(\alpha_{1} / \alpha_{2}\right)$, before decaying to 1.0 (when stimulation changes into inhibition) and finally to 0 as progressively more roots are infected (Fig. 1). The difference $\alpha_{1}-\alpha_{2}$ corresponds to the initial slope of $f(I)$ for a small number of infected roots and, therefore, determines the strength of stimulation. The parameter $\alpha_{2}$ describes the asymptotic decay of $f(I)$ for a large number of infected roots and, therefore, represents the level of inhibition.

Two wheat cultivars, Genghis and Savannah, were selected from a larger experiment as representative yet contrasting examples of the 20 cultivars that were screened for disease-induced root growth. Differences in epidemiological behavior between the two cultivars in response to infection by $G$. graminis var. tritici at low or high inoculum density were compared by (i) examining the rates of primary and secondary infection ( $r_{p}$ and $r_{s}$, respectively) and differences in the parameters for disease-induced root growth, $\alpha_{1}$ (primarily responsible for the stimulation levels) and $\alpha_{2}$ (determining the inhibition); (ii) comparison of curves describing change in the rate of disease-induced root production, $f$, with increase in numbers of infected roots; and (iii) estimation of differences in the duration of stimulated root production during the course of the epidemic.

The equations (2) were solved numerically and the solution used to estimate the parameters. The results were analyzed using both a conventional, frequentist approach and a Bayesian nonlinear estimation procedure involving Markov-chain Monte Carlo (MCMC) method. Here, we present results from the Bayesian framework because of the flexibility and computational ease of the MCMC technique (WinBUGS version 1.2, MRC Biostatistics Unit, Institute of Public Health, Cambridge, UK) and because this form of analysis has the advantage of a visual presentation of posterior densities for comparison of estimated parameters. We examined a range of priors representing various degrees of prior knowledge about the parameters. We used vague priors (uniform on the positive axis) rather than attempting to incorporate previous results $(2,4,5)$ in order to prevent bias associated with differ-

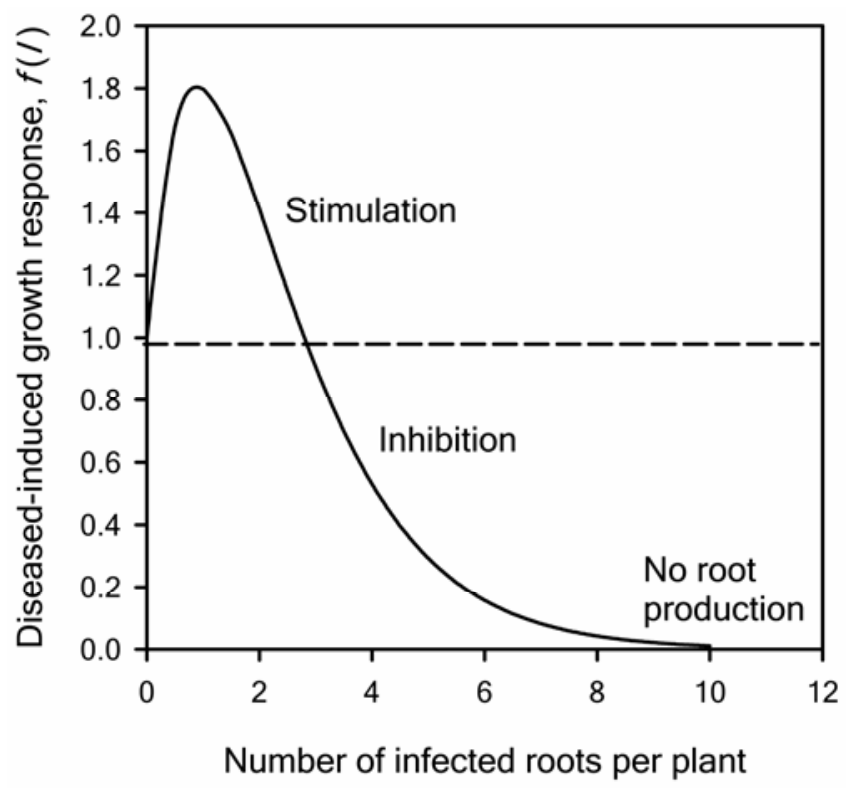

Fig. 1. Schematic representation of the response curve $f(I)=\left(1+\alpha_{1} I\right) \exp \left(-\alpha_{2} I\right)$, change in disease-induced root production with numbers of infected roots, where the parameter $\alpha_{1}$ determines the amplitude and the duration of stimulation and $\alpha_{2}$ determines the inhibition of root production. 
ent experimental conditions. We compared two alternative forms of the priors for the host response parameters $\left(\alpha_{1}\right.$ and $\left.\alpha_{2}\right)$; uniform priors on the positive axis represent a complete lack of prior knowledge, whereas an exponential distribution $P\left(\alpha_{i}\right)=$ $\lambda \exp \left(-\lambda \alpha_{i}\right)$ (for $i=1$ or 2 ) was used to explore the sensitivity of the posteriors to the prior choice. Exponential priors with a large value of $\lambda$ emphasize small values of the parameters $\alpha_{1}$, corresponding to a prior assumption that there is no host response. The estimators of the parameters were largely independent of the particular choice of the priors, except for very high values of $\lambda>$ 20 , when the prior is highly concentrated at 0 and conflicts with the likelihood. The likelihood was defined under an assumption of normal errors with variances fitted to the replicate data at each time point separately (these assumptions are equivalent to a weighted least-squares method). Two chains were run for 10,000 MCMC steps following a 1,000 burn-in period. Point estimates were obtained as medians for respective posterior distributions (Table 1; Figs. 2 and 3), and the limits for the high-density intervals were obtained as the respective 2.5 and $97.5 \%$ quantiles. The results of the Bayesian analysis were consistent with the equivalent frequentist approach.

Differences between posterior distributions corresponding to the parameters were quantified by calculating the Bhattacharyya coefficient $\gamma(9)$, equal to the sum of a square root of a product of

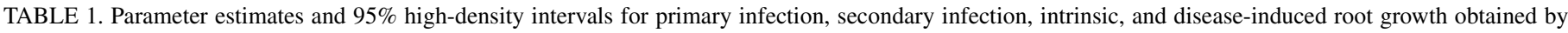

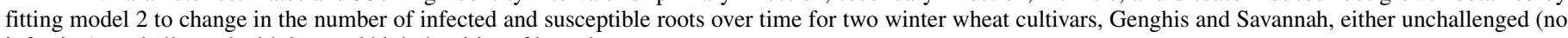
infection) or challenged with low and high densities of inoculum ${ }^{\mathrm{a}}$

\begin{tabular}{|c|c|c|c|c|c|c|}
\hline \multirow[b]{2}{*}{ Parameter $^{\mathrm{b}}$} & \multicolumn{3}{|c|}{ Genghis } & \multicolumn{3}{|c|}{ Savannah } \\
\hline & No infection & Low ID & High ID & No infection & Low ID & High ID \\
\hline$b$ & $0.025(0.021-0.033)$ & Fixed & Fixed & $0.023(0.020-0.030)$ & Fixed & Fixed \\
\hline $1 / \kappa$ & $0.017(0.001-0.042)$ & Fixed & Fixed & $0.013(0.001-0.039)$ & Fixed & Fixed \\
\hline$N_{0}$ & $3.1(2.8-3.4)$ & Fixed & Fixed & $3.1(2.9-3.4)$ & Fixed & Fixed \\
\hline$r_{p}$ & $\ldots$ & $0.057(0.043-0.074)$ & $0.038(0.025-0.050)$ & $\ldots$ & $0.044(0.032-0.057)$ & $0.023(0.018-0.029)$ \\
\hline$r_{s}$ & $\ldots$ & $0.010(0.009-0.012)$ & $0.015(0.011-0.021)$ & $\ldots$ & $0.014(0.012-0.016)$ & $0.015(0.013-0.017)$ \\
\hline$\alpha_{1}$ & $\ldots$ & $0.53(0.22-1.01)$ & $2.2(1.0-5.0)$ & $\ldots$ & $0.49(0.26-0.80)$ & $0.80(0.47-1.24)$ \\
\hline$\alpha_{2}$ & $\ldots$ & $0.29(0.15-0.47)$ & $0.61(0.41-0.87)$ & $\ldots$ & $0.21(0.12-0.30)$ & $0.31(0.22-0.41)$ \\
\hline
\end{tabular}

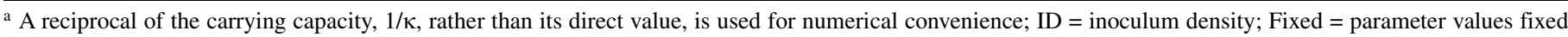
from estimates of intrinsic growth in the absence of disease.

b Parameters: $b=$ the per capita rate of production of susceptible roots, $N_{0}=$ the initial root number, $r_{p}=$ primary infection, and $r_{s}=$ secondary infection.
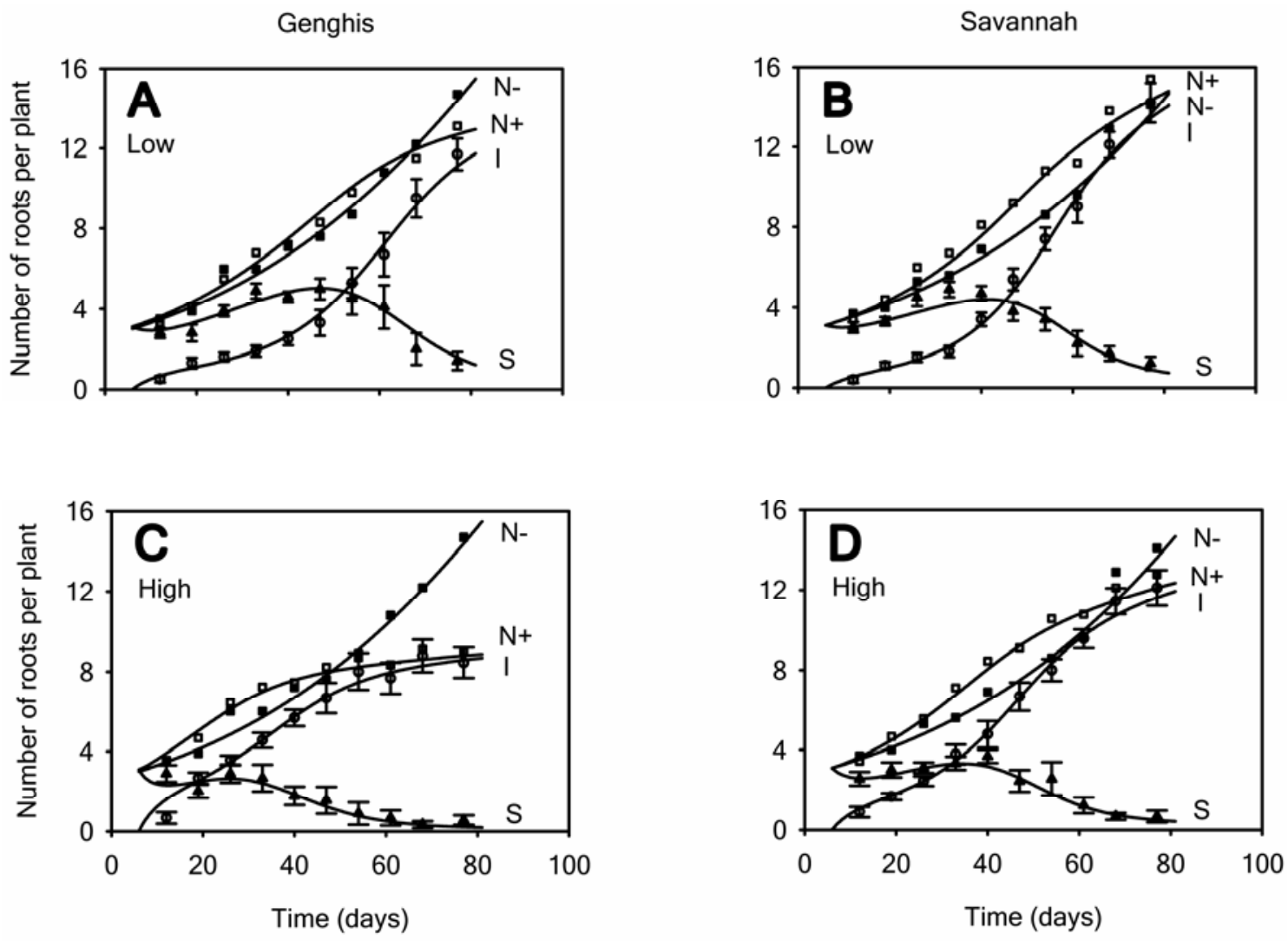

\section{$\mathrm{N}$ - Total roots, disease-free plants \\ I Infected roots, diseased plants \\ S Susceptible roots, diseased plants $\mathrm{N}+$ Total roots, diseased plants}

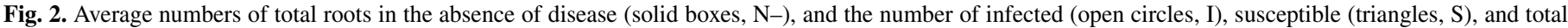

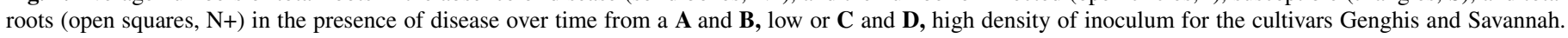
Curves represent fit of equation 2 to data (Table 1 provides parameter estimates). 
distributions $p_{1}(x)$ and $p_{2}(x)$. A perfect overlap of distributions (identical posterior distributions in both treatments, $p_{1}(x)=p_{2}(x)$ ) corresponds to $\gamma=1$ and low values of $\gamma$ correspond to a small area of overlap ( $\gamma=0$ for $p_{1}(x) p_{2}(x)=0$ everywhere).

Experimentation. Disease progress curves. Data for disease dynamics and host growth were obtained by growing 30 plants of each cultivar (Savannah and Ghengis) singly in replicated sand microcosms consisting of lengths of layflat tubing (Layflat Tubing, Isle of Wight) measuring $250 \mathrm{~mm}$ in length and $100 \mathrm{~mm}$ wide. Tubing was stapled at one end and filled with 1,000 g of sand (grade 16/30; Hepworth Minerals and Chemicals Ltd., Redhill, Surrey, UK). A nutrient solution (Hoagland's) was added to achieve a gravimetric water content of $10 \%$ by weight. Each microcosm was flattened to achieve a thickness of $\approx 20 \mathrm{~mm}$ and planted with a single pregerminated seed. Microcosms were incubated at $17^{\circ} \mathrm{C}$ with $16 \mathrm{~h}$ of light and grown on an incline of $30^{\circ}$ from vertical to guide roots to the rear of the pack. Inoculum was prepared by removing disks of colonized potato dextrose agar from the growing edge of a 7-day-old culture of G. graminis var. tritici (IMI 389305). After 6 days, either a single inoculum unit (low inoculum density) or four inoculum units (high inoculum density) were introduced into 10 of the microcosms at a depth of $100 \mathrm{~mm}$. This provided 10 microcosms with no inoculum, 10 with a low density of inoculum, and 10 with a high density of inoculum for each cultivar of wheat. A nondestructive, visual assessment (aided by a binocular microscope, $\times 20$ ) of the number of diseased (showing stelar discoloration) and disease-free main root axes was made each week for 10 consecutive weeks following inoculation. Roots were considered for counting when they had reached the front surface of the pack which, in almost all cases and because of the incline on which the plants were grown, would be at a distance of no more than $20 \mathrm{~mm}$ below the seed. Main root axes were distinguished from lateral roots on the basis of orientation and root width, confirmed by destructive sampling at the end of the experiment. Between observations, packs were covered with black polythene to avoid exposure of the roots to light. Soil packs were maintained at constant weight by adding Hoagland's nutrient solution (13).

Inoculum decay. To estimate the rate of inoculum decay, $r_{d}$, and hence the duration of primary infection, the decay in infectivity of inoculum was examined independently during the first 5 weeks of the experiment. Six units of inoculum were placed in each of 10 sand microcosms at the beginning of the experiment. After $0,6,10,14,18,28$, and 35 days, a single unit of inoculum was removed from each microcosm and placed against the seminal roots of a 7-day-old wheat plant (cv. Savannah). The sand packs were incubated under the conditions described above and assessed for disease after 21 days to provide estimates for change in the proportion of infective inoculum units with time.

\section{RESULTS}

Disease and host dynamics. Parameters for the decay of particulate inoculum were estimated from an independent experiment whereas parameters for intrinsic root growth were estimated from change in the number of roots over time in the absence of disease. Subsequently, their values were fixed in the model describing disease progress. Model 2 accurately described changes in the number of infected, susceptible, and total roots over time for all treatments (Fig. 2) and allowed estimation of the rates of primary infection, $r_{p}$, secondary infection, $r_{s}$, and values for disease-induced root growth, $\alpha_{1}$ and $\alpha_{2}$ (Table 1 ).

In the absence of disease, the cvs. Genghis and Savannah produced the same numbers of roots per plant over time. Root number increased from an average of 4 after 6 days to 15 after 80 days (Fig. 2, N-). When challenged with a low density of inoculum, the behavior of the two cultivars was only marginally different. The number of infected roots increased monotonically to an initial plateau of between one and two infected roots after 30 days for both Genghis and Savannah (Fig. 2A and B, I). This was followed by a rise for a further 50 days, after which 12 roots per plant, on average, were infected. During the same period, the number of susceptible roots increased to a maximum of approximately four per plant and then decayed to zero (Fig. 2A and B, S). Total root production increased initially for both cultivars of wheat (Fig. 2A and B, N+). However, although this increase was maintained for cv. Savannah (Fig. 2B, N+), for Genghis, the rate of root production had slowed so that, by the end of the epidemic,
Low
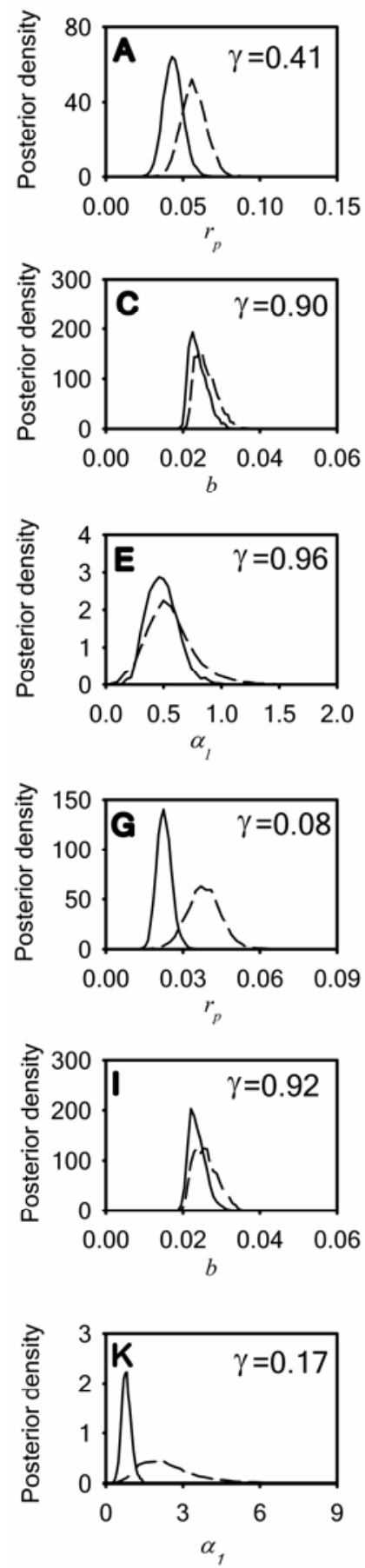

High
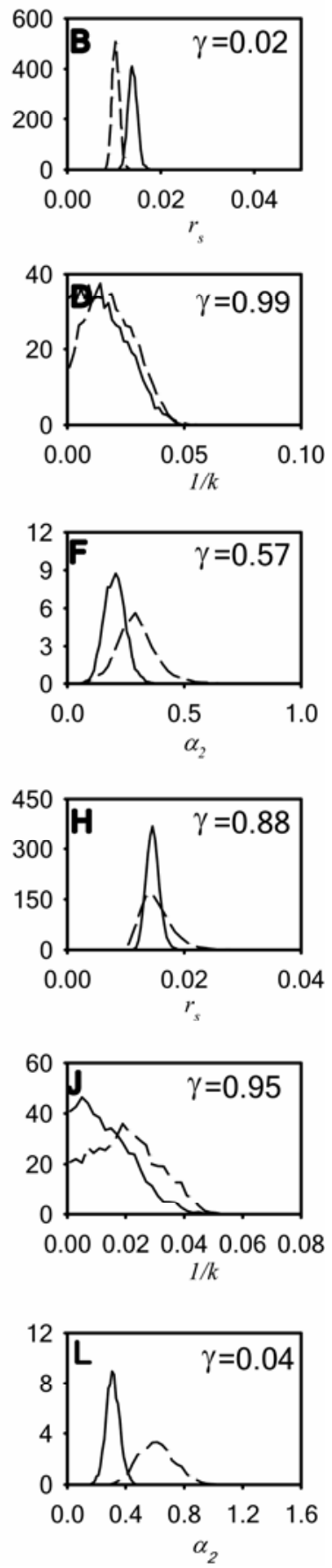

Fig. 3. Posterior distributions comparing estimates for the parameters $b, 1 / \kappa$, $r_{p}, r_{s}, \alpha_{1}$, and $\alpha_{2}$ for Genghis (broken lines) and Savannah (solid lines) from epidemics initiated from a $\mathbf{A}$ to $\mathbf{F}$, low or $\mathbf{G}$ to $\mathbf{L}$, high density of particulate inoculum. The similarity index, $\gamma$, reflects the probability that the parameters are identical ( $\gamma$ approaches 1$)$ or different $(\gamma$ approaches 0$)$. 
uninfected plants had produced more roots than infected plants (Fig. 2A, N+).

When challenged with a high density of inoculum, the difference between the two cultivars was more pronounced. The initial plateau increased to between four and five infected roots for Genghis and to between three and four for Savannah (Fig. 2C and $\mathrm{D}, \mathrm{I})$ whereas the number of susceptible roots increased only slightly and then decreased to zero (Fig. 2C and D, S). Total root production increased initially for both cultivars of wheat at a rate faster than that for intrinsic root production (Fig. 2C and D, N+). However, although the increase in root production for Savannah was maintained for 65 days and roots were still being produced by the end of the experiment (Fig. 2D, N+), for Genghis, the rate of root production had ceased after 50 days. This meant that, by the end of the epidemic, uninfected plants had produced, on average, six more roots than infected plants $(P=0.00002,17 \mathrm{df})$ (Fig. 2C, N+).

Estimation of parameters. Trends in the increase in root number over time in the absence of disease were described by a logistic curve with rate $b$ and a carrying capacity $\kappa$. The estimates of $1 / \kappa$ for the logistic model (Table 1 ) corresponded to the total number of 50 to 60 roots per plant, which was larger than expected but served to provide a limit to the potential growth. Thus, in the absence of disease, the production of roots was practically unlimited throughout the duration of the experiment. We also considered a simplified model assuming exponential growth corresponding to unlimited root growth, but found that all inferences based on the logistic model still held in the exponential case. Therefore, we decided to use the more realistic logistic model. No difference in either the carrying capacity $\kappa(\gamma=0.92)$ or the intrinsic rate of root production, $b(\gamma=0.85)$, was detected between the two wheat cultivars (Fig. 3). At low inoculum density, we detected no difference in rates of primary infection $\left(r_{p}\right)$ between Genghis and Savannah $(\gamma=0.41)$ (Table 1) but a significant increase in the rate of secondary infection, $r_{s}(\gamma=0.02)$ for Savannah. At high inoculum density, the rate of primary infection was lower for Savannah over Genghis $(\gamma=0.08)$ but no difference in secondary infection was detected $(\gamma=0.88)$. Differences between Genghis and Savannah were detected for parameters describing disease-induced root growth (Table 1). At low inoculum density, $\alpha_{1}$ and $\alpha_{2}$ were similar $\left(\gamma=0.96\right.$ for $\alpha_{1}$ and $\gamma=0.57$ for $\left.\alpha_{2}\right)$ but, at the high inoculum density, parameter estimates for both stimulation, $\alpha_{1}(\gamma=0.17)$, and inhibition, $\alpha_{2}(\gamma=0.04)$, of root production were higher for Genghis.

The shape of the estimated response curves, $f(I)$ (Fig. 4A and $B)$, reflect the differences in parameter estimates and, hence, differences in stimulation and inhibition of root production between the two cultivars when different numbers of roots are infected. We detected a phase of stimulation followed by inhibition for both cultivars at both densities of inoculum (Fig. 4A and B). For Savannah at a low density of initial inoculum, the amplitude of the increase was higher and extended over a wider range of infected roots than for cv. Genghis. The switch from stimulation to inhibition of root growth occurred when 5.0 and 9.0 roots were infected for Genghis and Savannah, respectively. From a high inoculum density, the amplitude of the stimulatory portion of the curve was higher but shorter for Genghis. The switch from stimulation to inhibition of root growth occurred when 4.5 and 6.7 roots were infected for Genghis and Savannah, respectively.

The differences in disease-induced root production, $f(I)$, with changes in numbers of infected roots were translated into curves describing change in disease-induced root production during the time course of the epidemic (Fig. 4C and D). At low inoculum density, Savannah maintained increased root production at a
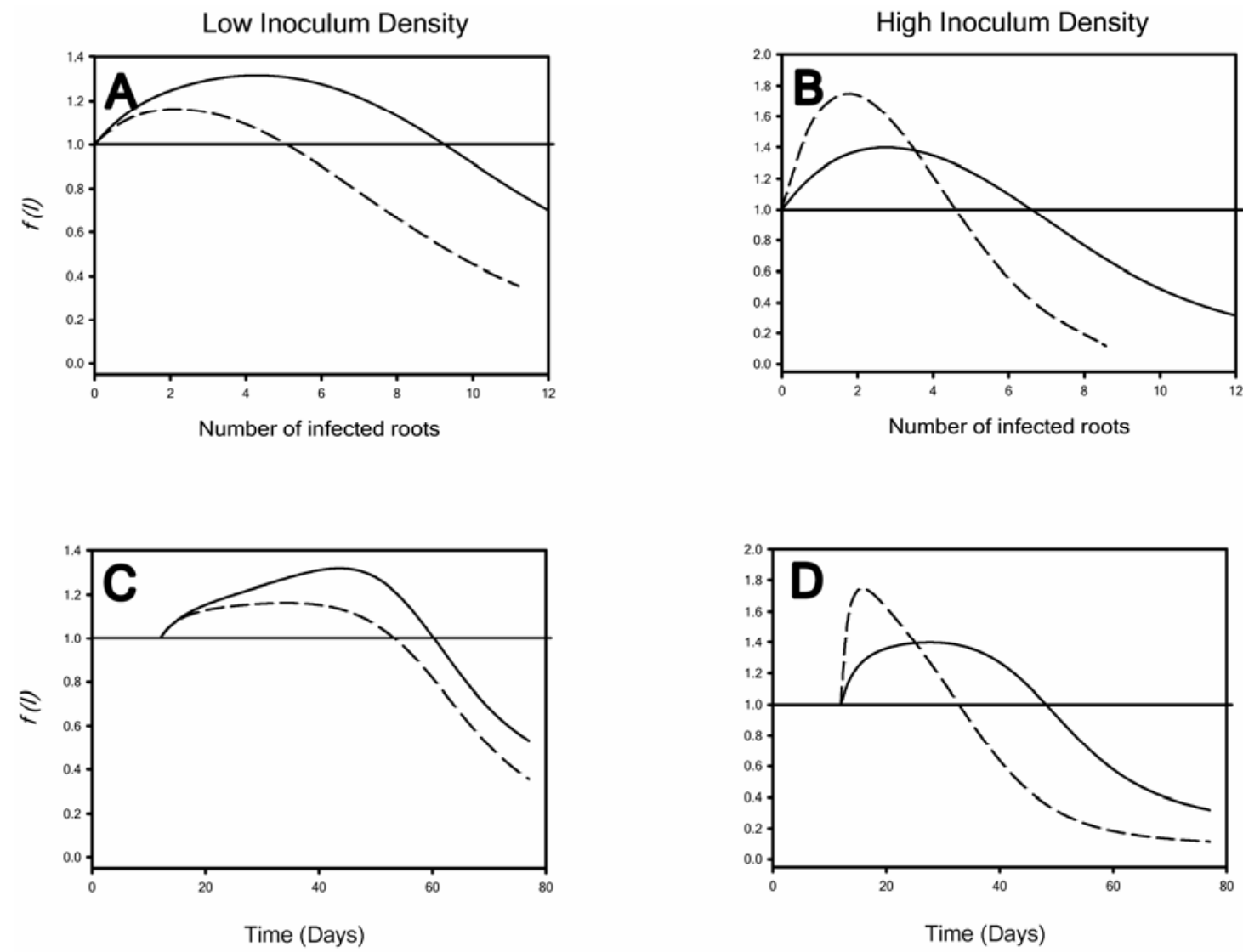

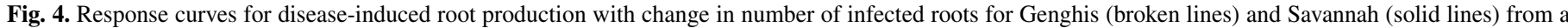

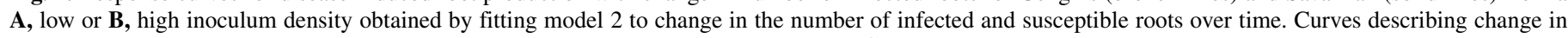
disease-induced root production, $f(I)$, over time are shown for epidemics initiated from a $\mathbf{C}$, low or $\mathbf{D}$, high inoculum density. 
higher rate and for longer than Ghengis. The switch between stimulation and inhibition occurred at 53 and 60 days for Genghis and Savannah, respectively. At high inoculum density, Genghis responded to disease with a high initial rate of root production but this lasted for only 33 days of the epidemic. In contrast, increased root production was detected for Savannah for a period of 48 days. Periods of root growth stimulation were followed by a period of root growth inhibition which caused the cessation of root production for Genghis after $\approx 60$ days. Root production for Savannah had not ceased by the end of the experiment.

\section{DISCUSSION}

In this article, we have used controlled environment experimentation and epidemiological modeling to compare the response of different wheat cultivars to infection by the take-all pathogen, G. graminis. The objective was to show proof of concept for combining experimentation and modeling to identify different host responses in root production among wheat cultivars to infection by $G$. graminis. To demonstrate this, two cultivars were selected for analysis as representative yet contrasting examples taken from a larger experiment. Although, in the absence of disease, root production was the same for Genghis and Savannah, we detected significant differences in behavior when infected by G. graminis var. tritici.

The effects were accentuated by increasing inoculum density. For Savannah, this was characterized by a reduction in the rate of primary infection, $r_{p}$, with no change in the rates of secondary infection, $r_{s}$, or disease-induced root growth, $\alpha_{1}$ and $\alpha_{2}$, suggesting a relatively high degree of tolerance to disease and broadly consistent with previous results (4). In contrast, for Genghis, increases in parameters for disease-induced root growth and in the rate of secondary infection, $r_{s}$, were detected, consistent with poor tolerance to increasing levels of disease pressure and leading to cessation of root growth by the end of the experiment.

That Savannah was able to stimulate root production for 15 days longer than Genghis at high inoculum density compared with only 7 days at low inoculum density (Fig. 4C and D) may reflect not only its enhanced properties of disease-induced root growth but also some degree of innate resistance to primary infection by $G$. graminis suggested by the reduced rate of primary infection. Likewise, for Genghis, the reduced threshold of disease-induced root growth may be somewhat offset by a reduction in the rate of secondary infection, perhaps in response to an increase in resistance or a difference in spatial architecture of the adventitious root system. Similar differences in innate resistance of seminal roots have been detected between cultivars as a consequence of increased thickening of cortical cell walls (17), whereas the spatial distribution of the host population is well known to affect rates of disease spread (5). It is plausible, then, to imagine that the improved performance of certain wheat cultivars during years of high risk to take-all are due not to a single, dominating factor but to combinations of factors, each making only a small contribution to the course of an epidemic and difficult to detect when tested in isolation, particularly in the presence of environmental heterogeneity (21). However, acting together or by better managing their behavior in combination with existing cultural and chemical forms of control, they may provide significant benefits in disease reduction and yield improvement during years of high-risk to take-all.

The successful detection and quantification of varietal differences in disease-induced root growth was achieved here by very careful control of other sources of variability: host germination, initial inoculum density, environmental conditions, and pathogen genotype. Differences in disease dynamics also could have developed, not only because of differences in the rate of primary infection, secondary infection, and intrinsic or disease-induced root growth, but as a result of differences in the infectivity of particulate inoculum. However, under such close environmental control, we can reasonably assume that the decay of inoculum was identical for both cultivars and not responsible for differences in epidemic development. Although the experiment was performed in a controlled environment, we selected a highly pathogenic isolate of G. graminis and optimal conditions for the spread of take-all representing epidemics that, together, gave rise to high disease severity even from a low initial inoculum density. In contrast, field epidemics are characterized by environmental heterogeneity and variation in isolate pathogenicity. This will create regions within a field, suboptimal for the spread of disease with the potential to extend the period of root stimulation. Moreover, field epidemics are further characterized by variability in the distribution of initial inoculum at the time of planting (15). This alone could create differences in the dynamics of the response for the two cultivars tested (Fig. 4). Add to this the heterogeneous nature of the soil environment and variability in the pathogenicity of different strains of G. graminis, and it is little wonder that such mechanisms as disease-induced host growth go undetected in field experimentation.

When compared with other cereal crops, the tolerance of wheat to infection by $G$. graminis var. tritici is not high (14). Yet even a small improvement in tolerance during years of high disease risk may provide a significant economic benefit. The complexity of the take-all system is almost certainly complicated by interactions with the environment whereby the benefits of growing more tolerant cultivars may well depend on the relative contributions of primary and secondary infection. We propose that the epidemiological approach presented here can be extended to tease out and understand the roles of multiple epidemiological factors involved in the improved performance of certain wheat cultivars to take-all and, ultimately, to assist in the screening of plant cultivars for better disease tolerance and in the provision of guidelines for plant design aimed at better disease management.

\section{ACKNOWLEDGMENTS}

This work was co-funded by an award from the Biotechnology and Biological Sciences Research Council and by CPB Twyfords, U.K., which we gratefully acknowledge.

\section{LITERATURE CITED}

1. Anderson, R. M., and May, R. M. 1991. Infectious Diseases of Humans: Dynamics of Control. Oxford University Press, Oxford.

2. Asher, M. J. C. 1972. Effect of Ophiobolus graminis infection on the growth of wheat and barley. Ann. Appl. Biol. 70:215-223.

3. Bailey, D. J., and Gilligan, C. A. 1999. Dynamics of primary and secondary infection in take-all epidemics. Phytopathology 89:84-91.

4. Bailey, D. J., and Gilligan, C. A. 2004. Modeling and analysis of diseaseinduced host growth in the epidemiology of take-all. Phytopathology 94:535-540.

5. Bailey, D. J., Otten, W., and Gilligan, C. A. 2000. Percolation, heterogeneity and the saprotrophic invasion of soil by the fungal plant pathogen Rhizoctonia solani. New Phytol. 146:535-544.

6. Bailey, D. J., Paveley, N. A., Pillinger, C., Foulks, J., Spink, J, and Gilligan, C. A. 2005. Epidemiology and control of take-all on seminal and crown roots of wheat. Phytopathology 95:62-68.

7. Colbach, N., Lucas, P., and Meynard, J. M. 1997. Influence of crop management on take-all development and disease cycles on winter wheat. Phytopathology 87:26-32.

8. Cook, R. J. 2003. Take-all of wheat. Physiol. Mol. Plant Pathol. 62:73-86.

9. Fukunaga, K. 1990. Introduction to Statistical Pattern Recognition, 2nd ed. Academic Press, Boston.

10. Gilligan, C. A. 1994. The dynamics of infection by the take-all fungus on seminal roots of wheat-sensitivity analysis of a stochastic simulationmodel. New Phytol. 128:539-553.

11. Gilligan, C. A. 2002. An epidemiological framework for disease management. Adv. Bot. Res. 38:1-64.

12. Gilligan, C. A., Gubbins, S., and Simons, S. A. 1997. Analysis and fitting of an SIR model with host response to infection load for a plant disease. Philos. Trans. R. Soc. Lond. Ser. B Biol. Sci. 352:353-364.

13. Hoagland, D. R., and Arnon, D. I. 1950. The water culture method for growing plants without soil. In: Circular 347. California Agricultural 
Experiment Station, College of Agriculture, University of California, Berkeley.

14. Hollins, T. W. 1986. The relative resistance of wheat, rye and triticale to take-all caused by Gaeumannomyces graminis. Plant Pathol. 35:93-100.

15. Hornby, D. 1975. Inoculum of the take-all fungus-nature, measurement, distribution and survival. Eur. Mediterr. Plant Prot. Organ. 5:319-333.

16. Otten, W., Filipe, J. A. N., Bailey, D. J., and Gilligan, C. A. 2003. Quantification and analysis of transmission rates for soilborne epidemics. Ecology 84:3232-3239.

17. Penrose, L. 1987. Thickening and browning of cortical cell-walls in seminal roots of wheat seedlings infected with Gaeumannomyces graminis var. tritici. Ann. Appl. Biol. 110:463-470.

18. Penrose, L. D. J. 1994. Resistance to Gaeumannomyces graminis in wheat genotypes grown in field environments and sand culture. Soil Biol.
Biochem. 26:719-726.

19. Penrose, L. D. J. 1995. Two wheat genotypes differ in root disease due to Gaeumannomyces graminis without interaction with site. Soil Biol. Biochem. 27:133-138.

20. Schoeny, A., and Lucas, P. 1999. Modeling of take-all epidemics to evaluate the efficacy of a new seed-treatment fungicide on wheat. Phytopathology 89:954-961.

21. Scott, P. R. 1981. Variation in host susceptibility. Pages 219-236 in: Biology and Control of Take-All. M. J. C. Asher and P. J. C. Shipton, eds. Academic Press, New York.

22. Skou, J. P. 1975. Studies on the take-all fungus Gaeumannomyces graminis V. Development and regeneration of roots in cereal species during the attack. Kongelige Veterinaer-og Landbohøiskoles Aarsskrift $142-160$ 